\title{
Ferromagnetic Resonance in Metallic Thin Films and Thin-Film Tubes
}

\author{
J. DuBowIK \\ Institute of Molecular Physics, Polish Academy of Sciences \\ of Sciences \\ Smoluchowskiego 17, 60-179 Poznań, Poland \\ AND I. GoŚCIAŃSKA \\ Department of Physics, A. Mickiewicz University \\ Umultowska 85, 61-614 Poznań, Poland \\ A model describing ferromagnetic resonance in thin-film magnetic \\ metallic tubes is proposed and compared with the experimental ferromag- \\ netic resonance spectra of the thin-film $\mathrm{Ni}$.
}

PACS numbers: 75.70.-i, 75.30.Gw, 76.50.+g

\section{Introduction}

Ferromagnetic resonance (FMR) is a useful method in studying thin magnetic films (Fig. 1a). Using FMR the essential parameters describing magnetic properties of thin films can be measured: the effective magnetization $4 \pi M$, the

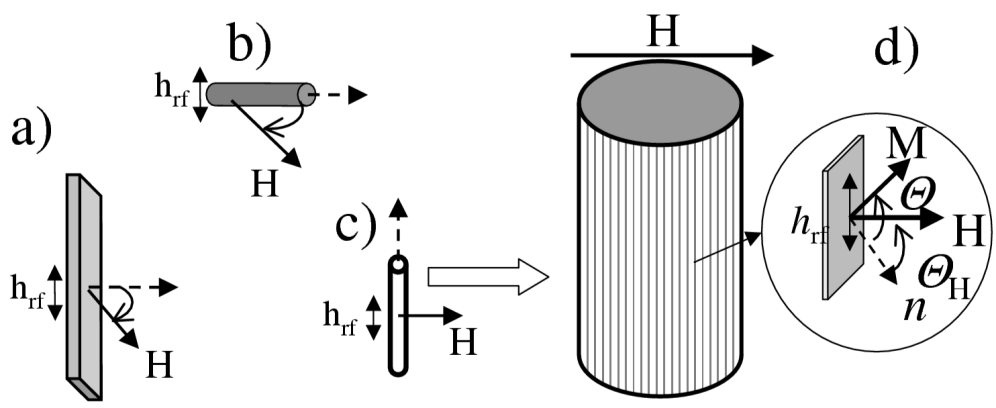

Fig. 1. Schematic drawings of the magnetic field configuration used in FMR measurements of thin films (a), magnetic wires (b), and thin-film tubes (c). Thin-film tube is divided into a large number of ribbons (d). Each ribbon is approximated by a thin film. 
spectroscopic splitting factor $g$ [1]. In magnetic wires (whiskers, amorphous wires or the arrays of nanowires) FMR has been also extensively investigated [2-4]. In "macroscopic" wires (Fig. 1b) of several $\mu \mathrm{m}$ in diameter, due to a finite skin-depth $\delta$, the resonance field (measured in a configuration shown in Fig. 1b) is a function of the ratio $a / \delta$, where $a$ is the wire diameter [3] and it has been shown to increase monotonically with $a / \delta$ from $H=\omega / \gamma-2 \pi M$ - typical of non-conducting cylinder, to the in-plane resonance field of metal films: $(\omega / \gamma)^{2}=H(H+4 \pi M)$. Similar results have been obtained for a system of $\mathrm{Ni}$ nanowire arrays $[4,5]$. Definitely, less attention has been paid to FMR in thin-film tubes, in which in opposite to the wires, the thickness of the magnetic film is limited to several nm (Fig. 1c) and skin-depth effect can be neglected. In the present contribution we show a model describing FMR in thin-film tubes and we compare the model with experimental results for $\mathrm{Ni}$ deposited onto glass rods and and the $\mathrm{Ni}-\mathrm{Mn}-\mathrm{Ga}$ thin film tubes.

\section{A model and comparison with the experiment}

FMR response in metallic thin-film tubes can be described in similar way as in thin films. We apply here a configuration of the magnetic fields (Fig. 1c and d), in which $h_{\mathrm{rf}}$ is applied parallel to the tube axis and the static magnetic field $H$ is applied perpendicular to it. Such a configuration has never been treated yet. Let us assume that the thin-film tube consists of many narrow thin-film ribbons as it is shown in Fig. 1d. Each ribbon is treated, in the first approximation, as a "typical" thin film with the normal $n$ to its plane making an angle $\Theta_{H}$ with the field $H$. For the entire tube, the individual ribbons have their normals $n$ oriented with respect to the field $H$ in a range of $0 \leq \Theta_{H} \leq 2 \pi$. For an individual ribbon the resonance conditions for the uniform mode are described by a standard relation [1]:

$$
\begin{gathered}
\left(\frac{\omega}{\gamma}\right)^{2}=\left[H_{\mathrm{r}} \cos \left(\Theta-\Theta_{H}\right)-4 \pi M \cos 2\left(\Theta-\Theta_{H}\right)\right] \\
\times\left[H_{\mathrm{r}} \cos \left(\Theta-\Theta_{H}\right)-4 \pi M \cos ^{2}\left(\Theta-\Theta_{H}\right)\right]
\end{gathered}
$$

with equilibrium condition $H_{\mathrm{r}} \sin \left(\Theta-\Theta_{H}\right)=4 \pi M \sin \Theta \cos \Theta$ for magnetization $M$ oriented at an angle $\Theta$ with respect to normal $n$. $\omega=2 \pi f$ is the frequency of microwaves, $\gamma$ is gyromagnetic ratio and $M$ is the effective magnetization.

To obtain a FMR response of the entire thin-film tube, we can treat, in the first approximation, the set of our thin-film ribbons as independent in a similar way as the FMR in ferrite powders has been treated in "independent grain approach" [6]. Equation (1) is calculated for a large number of $\Theta_{H}$ values covering the range $0-2 \pi$ and the results are historammed on a magnetic field scale. Figure 2a shows a calculated histogram which actually represents the "density" of the reduced resonance fields $h_{\mathrm{r}}=H_{\mathrm{r}} /(\omega / \gamma)$ in the range $0 \leq \Theta_{H} \leq 2 \pi$ for infinitesimally narrow resonance line width. To simulate an experimental spectrum of a thin-film tube, the histogram $f_{k}$ is convoluted with an appropriate Lorentzian broadening function $L_{j, k}$ 

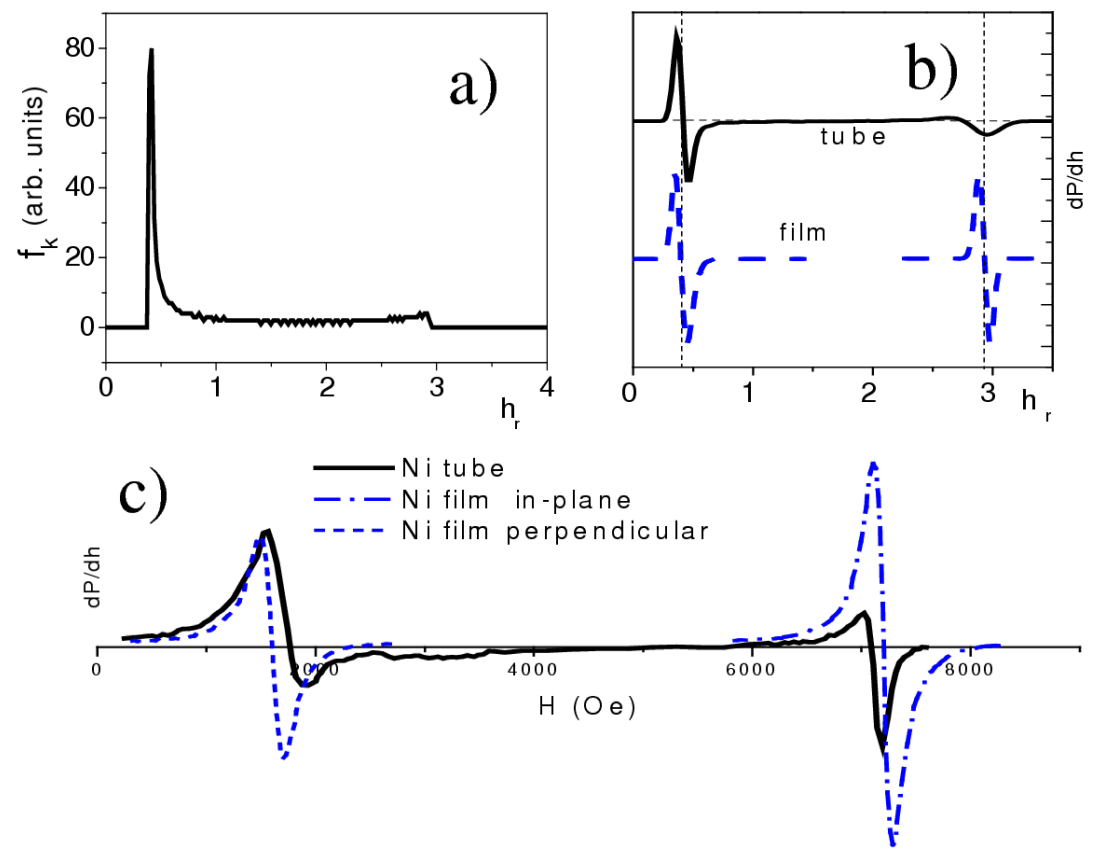

Fig. 2. (a) A histogram showing FMR pattern of a system of ribbons forming a thinfilm tube. (b) Calculated first-derivative FMR spectra of a tube for finite line broadening (continuous curve) Dashed curves show the calculated spectra for a thin film. (c) Experimental spectra for a Ni thin-film tube (continuous curve) taken in the configuration shown in Fig. 1c. and for a Ni thin film (dashed curves) taken at the in-plane and perpendicular to the plane configuration, respectively.

$$
L_{j}=\sum L_{j, k} f_{k},
$$

and the first derivative of the broadened absorption curve $L_{j}$ is taken.

Figure 2b (continuous curve) shows FMR spectrum calculated for a hypothetical Ni thin-film tube $(M=485 \mathrm{Gs}, g=2.2)$ and, for a comparison, the spectra of a "normal" thin film (dashed curves) taken at $\Theta_{H}=0^{\circ}$ and $90^{\circ}$, respectively. The most characteristic in FMR response of thin-film tubes is that the "peaks" at the shoulders are asymmetric but nearly correspond to spectra of the thin film. HH

Figure 2c shows experimental FMR spectra of a $100 \mathrm{~nm}$ Ni film (dashed curves) taken at in-plane $(\|)$ and perpendicular to the plane $(\perp)$ configurations, respectively. The resonance fields $H_{\mathrm{r} \|}=1310$ Oe and $H_{\mathrm{r} \perp}=8200$ Oe give $g=2.21$ typical of $\mathrm{Ni}$ and $4 \pi M=5265 \mathrm{Gs}$, i.e., slightly lower than 6050 Gs for pure $\mathrm{Ni}$. The continuous curve in Fig. 2c shows the FMR spectrum of the thin-film Ni tube. It is seen that the the low- and high-field peaks situated on the shoulders of the spectrum approximately coincide with the Ni film's spectra taken at the in-plane and perpendicular to the plane configurations, respectively. Hence, the 
FMR results for the thin-film Ni tube are qualitatively consistent with our simple model. Such an approach has been proved useful to study magnetic properties of thin-film tubes obtained from thin Ni-Mn-Ga films after separation from the mica substrates [7]. Ni-Mn-Ga alloy exhibits large strains due to magnetic field induced rearrangement of the martensite variants [8]. This effect is diminished in thin films due to constraint of a substrate. Therefore, we were interested in the magnetic behavior of the films released from the substrates.

\section{Summary}

In conclusion, the FMR in thin-film metallic tubes has been qualitatively modelled in the framework of "independent ribbon" approach.

\section{Acknowledgments}

The work was sponsored by the State Committee for Scientific Research grant No. 4 T08C 04525.

\section{References}

[1] M. Farle, Rep. Prog. Phys. 61, 755 (1998).

[2] B. Heinrich, Czech. J. Phys. B 17, 142 (1967).

[3] M. Maryško, Phys. Status Solidi A 47, 277 (1978).

[4] U. Ebels, J.-L. Duvail, P.E. Wigen, L. Piraux, L.D. Buda, K. Ounadjela, Phys. Rev. B 64, 144421 (2001)

[5] R. Arias, D.L. Mills, Phys. Rev. B 67, 094423 (2003).

[6] E. Schlomann, J. Phys. Chem. Solids 6, 257 (1958).

[7] J. Dubowik, I. Gościańska, J. Magn. Magn. Mater. 316, e599 (2007).

[8] K. Ullakko, J.H. Huang, C. Kantener, R.C. O'Handley, V.V. Kokorin, Appl. Phys. Lett. 69, 1966 (1996). 